\title{
Enantioselective Desymmetrization of FTY720
}

\author{
Hidemasa Hikawa,* Maiko Hamada, Yuka Uchida, Shoko Kikkawa, Yuusaku Yokoyama,* and \\ Isao Azumaya
}

Faculty of Pharmaceutical Sciences, Toho University; 2-2-1 Miyama, Funabashi, Chiba 274-8510, Japan.

Received June 20, 2014; accepted July 24, 2014

\begin{abstract}
A method for enantioselective desymmetrization of $N$-Ac and $N$-Boc-FTY720 by nonenzymatic asymmetric acylation was developed. Effective enantioselective monobenzoylation using benzoyl chloride in the presence of the tetraphenylbisoxazoline $(\mathrm{L} 2)-\mathrm{CuCl}_{2}$ complex gave the desired products $3 \mathrm{a}$ and $3 \mathrm{~b}$ in $52-62 \%$ yield with $64 \%$ ee.
\end{abstract}

Key words desymmetrization; FTY720; $\mathrm{CuCl}_{2}$; asymmetric synthesis; tetraphenylbisoxazoline

FTY720 (Fingolimod) is a first-in-class orally bioavailable compound that has shown efficacy in advanced clinical trials for the treatment of multiple sclerosis (MS), and has already been approved in the U.S., Russia, Switzerland, Australia and the EU as a first-line treatment for relapsing forms of MS. FTY720 is the first of a novel class of sphingosine-1-phosphate (S1P) receptor modulators ${ }^{1-3)}$ (Fig. 1), and is rapidly monophosphorylated in vivo to form FTY720-phosphate, which is an agonist for four sphingosine-1-phosphate (S1P) receptors $\left(\mathrm{S}_{1} \mathrm{P}_{1,3,4,5}\right)$. Notably, although FTY720 was designed by deleting the asymmetric centers of its lead compound, FTY720phosphate has an asymmetric carbon. ${ }^{4-8)}$ Therefore, a practical asymmetric synthesis of both enantiomers of the immunosuppressive FTY720-phosphate is essential for pharmacological evaluation. Recently, efficient enzymatic asymmetric acylation of $N$-protected FTY720 has been reported. Kiuchi et al. reported the asymmetric synthesis of FTY720-phosphate using lipase-catalyzed acylation as a key step. ${ }^{3)}$ In contrast, to the best of our knowledge, nonenzymatic enantioselective acylation of FTY720 derivatives has not been described before.

Enantioselective desymmetrization of symmetric molecules has been investigated for constructing versatile chiral building blocks, and is an economical way to generate chirality. Re-<smiles>NC(CO)(CO)CCc1ccc(CCC(N)(CO)COP(=O)(O)O)cc1</smiles>

Fig. 1. FTY720 and FTY720-Phosphate

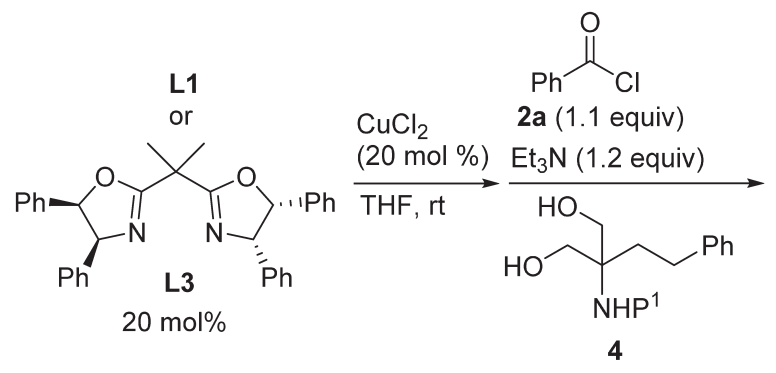

cently, much attention has been focused on the nonenzymatic asymmetric acylation of prochiral and meso diols. While catalytic asymmetric desymmetrization methods for meso 1,2diols have been reported, ${ }^{9-11)}$ prochiral 1,3-diols are extremely rare. $^{12-17)}$

Herein, we report the first example of nonenzymatic enantioselective monobenzoylation of $N$-Ac and $N$-Boc-FTY720. Asymmetric synthesis using benzoyl chloride in the presence of tetraphenylbisoxazoline ( $\mathbf{L 2})-\mathrm{CuCl}_{2}$ complex gave the desired product 3. We have been developing this method for the selective monobenzoylation of FTY720 analogs. Investigation of the acylation of FTY720 analogs will provide new insights into the phosphorylation mechanism of sphingosine kinase. Most recently, we reported the selective monobenzoylation of $N$-Bz-FTY720 using benzoyl methyl phosphate as an efficient reagent. $^{18)}$

\section{Results and Discussion}

First, we stirred a mixture of $N$-Ac-FTY720 1a, benzoyl chloride 2a (1.1 eq), $\mathrm{CuCl}_{2}$ (1 eq) and diphenylbisoxazoline (L1) in tetrahydrofuran (THF) at room temperature for $2 \mathrm{~h}$. The desired monobenzoylated 3a was obtained in 44\% yield with $49 \%$ ee (entry 1). The reaction of $N$-Boc-FTY720 $\mathbf{1 b}$ with benzoyl chloride $\mathbf{2 a}$ resulted in almost the same enantioselectivity (entry 2, 51\% ee). With regard to the acylating agents, the use of $\mathrm{Ac}_{2} \mathrm{O}$ instead of benzoyl chloride $\mathbf{2 a}$ resulted in $38 \%$ yield with only $30 \%$ ee (entry 3 ). Furthermore, when using $\mathrm{CH}_{2} \mathrm{Cl}_{2}$, the reaction resulted in a lower ee value (entry 4, $12 \%$ ee; entry $5,8 \%$ ee). $\mathrm{AcCl}$ was ineffective (entry $6,0 \%$ ee). With regard to the box ligands, the best result was obtained when tetraphenylbisoxazoline (L2) was used instead of

Chart 1. Desymmetrization of Serinol $4^{15}$

The authors declare no conflict of interest. 
Table 1. Effect of Catalysts and Solvents on the Desymmetrization of $\mathbf{1}$ with Acylating Agents $\mathbf{2}^{a)}$
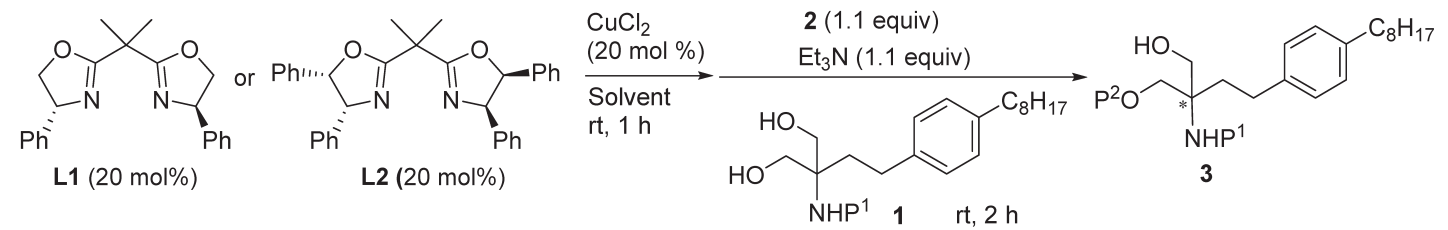

\begin{tabular}{|c|c|c|c|c|c|c|c|c|}
\hline Entry & Ligand & $P^{1}$ & Solvent & Reagent 2 & $P^{2}$ & Product & Yield $(\%)^{b)}$ & ee $(\%)$ \\
\hline 1 & L1 & Ac (1a) & THF & $\mathrm{BzCl}(\mathbf{2 a})$ & $\mathrm{Bz}$ & $3 \mathbf{a}$ & 44 & 49 \\
\hline 2 & L1 & Boc (1b) & THF & $\mathrm{BzCl}(\mathbf{2 a})$ & $\mathrm{Bz}$ & $3 b$ & 24 & 51 \\
\hline 3 & L1 & Ac (1a) & THF & $\mathrm{Ac}_{2} \mathrm{O}(\mathbf{2} \mathbf{b})$ & Ac & $3 c$ & 38 & 30 \\
\hline 4 & L1 & Ac (1a) & $\mathrm{CH}_{2} \mathrm{Cl}_{2}$ & $\mathrm{Ac}_{2} \mathrm{O}(\mathbf{2 b})$ & Ac & $3 c$ & 50 & 12 \\
\hline 5 & L1 & Boc (1b) & $\mathrm{CH}_{2} \mathrm{Cl}_{2}$ & $\mathrm{Ac}_{2} \mathrm{O}(\mathbf{2 b})$ & Ac & 3d & 48 & 8 \\
\hline 6 & L1 & Ac (1a) & THF & $\mathrm{AcCl}(2 \mathrm{c})$ & Ac & $3 c$ & 21 & 0 \\
\hline $7^{c)}$ & L2 & Ac (1a) & THF & $\mathrm{BzCl}(\mathbf{2 a})$ & $\mathrm{Bz}$ & $3 \mathbf{a}$ & 62 & 64 \\
\hline $8^{c)}$ & L2 & Boc (1b) & THF & $\mathrm{BzCl}(\mathbf{2 a})$ & $\mathrm{Bz}$ & $3 b$ & 52 & 64 \\
\hline
\end{tabular}

a) Reaction conditions: 1) $\mathrm{CuCl}_{2}(20 \mathrm{~mol} \%)$, $\mathbf{L} 1$ or $\mathbf{L 2}(20 \mathrm{~mol} \%)$, solvents (10 mL), rt, 1 h. 2) $\mathbf{1}(0.5 \mathrm{mmol}), \mathbf{2}$ (1.1 eq), Et ${ }_{3} \mathrm{~N}$ (1.1 eq), solvents (10 mL), rt, 2 h. b) Yield of isolated product. c) $24 \mathrm{~h}$.
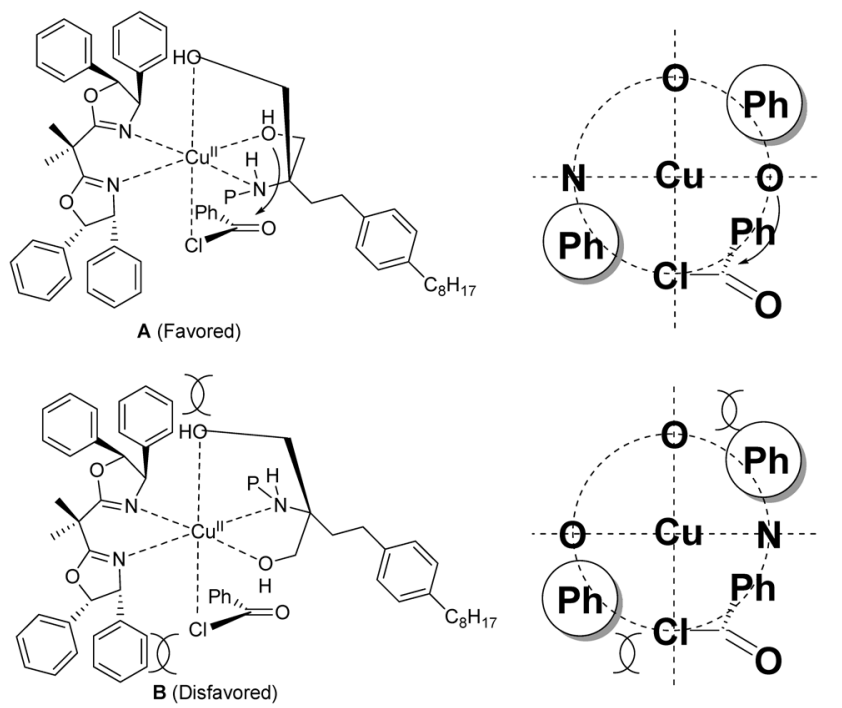

Fig. 2. Proposed Transition State Models

L1 (entries 7 and 8, 64\% ee).

Kang and colleagues reported the enantioselective formation of tert-alkylamines with benzoyl chloride by desymmetrization of 2-substituted serinols using $\mathrm{Box}-\mathrm{CuCl}_{2}$ complexes. ${ }^{15}$ ) The best desymmetrization was achieved with the benzamide diol using the $\mathbf{L 3}-\mathrm{CuCl}_{2}$ catalyst (94\% yield with $92 \%$ ee), while the reaction of $\mathrm{N}$-Boc serinol with $\mathbf{L} \mathbf{1}-\mathrm{CuCl}_{2}$ catalyst resulted in moderate stereoselection (only 6\% yield with $49 \%$ ee) (Chart 1). However, to achieve a practically efficient protocol, $\mathrm{N}$-Ac or $\mathrm{N}$-Boc groups must be cleaved under mild conditions, while harsh conditions are required for $N$-benzoyl deprotection. ${ }^{2,3,19-26)}$ Therefore, the use of protecting groups such as acetyl or Boc groups needs further development for enantioselective desymmetrization of $N$-protected FTY720. Additionally, the yield of acylation was increased by introduction of the $n$-octyl group on the benzene ring (entry 2 in Table 1 vs. Chart 1). Therefore, the hydrophobic properties of diols $\mathbf{1}$ might be important in this catalytic system (1b: $c \log P$ value of $6.9 ; 4 \mathbf{a}: c \log P$ value of 2.7$)$.

On the basis of our results and literature reports by Kang and colleagues, ${ }^{15)}$ an attempt was made to formulate a transi- tion state model to explain the stereochemical outcome of the enantioselective reaction (Fig. 2). First, the $\mathrm{Cu}$ (II) was ligated with both $N$-protected FTY720 1 as a tridentate ligand and Box ligand to form a pyramidal-shaped complex, and benzoyl chloride coordinated to $\mathrm{Cu}$ (II) from the bottom face to form intermediate $\mathbf{A}$ or $\mathbf{B}$. The desymmetrization of the 1,3-diol through complex $\mathbf{B}$ is disfavored since $\mathbf{B}$ is in a higher energy state due to steric interactions between the phenyl ring of benzoyl chloride and the phenyl ring in the oxazoline ring. Indeed, using $\mathrm{Ac}_{2} \mathrm{O}$ or $\mathrm{AcCl}$, which are less bulky molecules, was ineffective (see entries 3-6 in Table 1).

\section{Conclusion}

In summary, we have developed the first method for enantioselective desymmetrization of $N$-Ac and $N$-Boc-FTY720 1 by nonenzymatic asymmetric acylation. Effective desymmetrization was achieved using benzoyl chloride (2a) and triethylamine in the presence of tetraphenylbisoxazoline (L2) $-\mathrm{CuCl}_{2}$ complex in THF at room temperature to give the desired $\mathbf{3 a}, \mathbf{b}$ in $52-62 \%$ yield with $64 \%$ ee. Additionally, protecting groups such as acetyl or Boc groups could be used in the enantioselective desymmetrization.

\section{Experimental}

2-Acetamido-2-(hydroxymethyl)-4-(4-octylphenyl)butyl Benzoate 3a (Entry 7) To a solution of tetraphenylbisoxazoline (L2) $(24 \mathrm{mg}, 0.05 \mathrm{mmol})$ in THF $(2.5 \mathrm{~mL})$ was added $\mathrm{CuCl}_{2}(6.7 \mathrm{mg}, 0.05 \mathrm{mmol})$ and stired at room temperature for $1 \mathrm{~h}$. To the mixture was added THF $(5.0 \mathrm{~mL}), N$-(1-hydroxy-2(hydroxymethyl)-4-(4-octylphenyl)butan-2-yl)acetamide $(87 \mathrm{mg}, 0.25 \mathrm{mmol}), \mathrm{Et}_{3} \mathrm{~N}(0.37 \mathrm{~mL}, 0.26 \mathrm{mmol})$, benzoyl chloride $(0.23 \mathrm{~mL}, 0.26 \mathrm{mmol})$ were added. After being stirred at room temperature for $24 \mathrm{~h}$, the mixture was poured into water and extracted with EtOAc. The organic layer was washed with brine, dried over $\mathrm{Na}_{2} \mathrm{SO}_{4}$, and concentrated in vacuo. The residue was purified by silica gel column chromatography (hexane-EtOAc) to give $\mathbf{3 a}(70 \mathrm{mg}, 62 \%)$ as a colorless oil.

IR (KBr) $\left(\mathrm{cm}^{-1}\right)$ 3296, 1719, 1650; ${ }^{1} \mathrm{H}-\mathrm{NMR}(400 \mathrm{MHz}$, $\left.\mathrm{CDCl}_{3}\right) \delta: 0.87(\mathrm{t}, J=6.9 \mathrm{~Hz}, 3 \mathrm{H}), 1.20-1.35(\mathrm{~m}, 10 \mathrm{H}), 1.57$ (quin., $J=7.3 \mathrm{~Hz}, 2 \mathrm{H}$ ), 1.95 (s, 3H), 2.04 (ddd, $J=14.2,11.5$, $6.0 \mathrm{~Hz}, 1 \mathrm{H}), 2.27$ (ddd, $J=14.2,11.0,5.5 \mathrm{~Hz}, 1 \mathrm{H}), 2.55(\mathrm{t}$, 
$J=7.8 \mathrm{~Hz}, 2 \mathrm{H}), 2.61$ (ddd, $J=13.5,11.2,5.5 \mathrm{~Hz}, 1 \mathrm{H}), 2.71$ (ddd, $J=13.5,11.2,5.5 \mathrm{~Hz}, 1 \mathrm{H}), 3.80$ (d, $J=12.1 \mathrm{~Hz}, 1 \mathrm{H}), 3.84$ (d, $J=12.1 \mathrm{~Hz}, 1 \mathrm{H}), 4.48(\mathrm{~d}, J=11.5 \mathrm{~Hz}, 1 \mathrm{H}), 4.62(\mathrm{~d}, J=11.5 \mathrm{~Hz}$, $1 \mathrm{H}), 5.89$ (s, 1H), $7.08(\mathrm{~d}, J=8.5 \mathrm{~Hz}, 2 \mathrm{H}), 7.11(\mathrm{~d}, J=8.5 \mathrm{~Hz}$, $2 \mathrm{H}), 7.47$ (dd, $J=8.0,1.6 \mathrm{~Hz}, 2 \mathrm{H}), 7.60(\mathrm{tt}, J=7.3,1.4 \mathrm{~Hz}, 1 \mathrm{H})$, $8.03(\mathrm{dd}, J=7.3,1.4 \mathrm{~Hz}, 2 \mathrm{H}) ;{ }^{13} \mathrm{C}-\mathrm{NMR}\left(100 \mathrm{MHz}, \mathrm{CDCl}_{3}\right) \delta$ : $14.1,22.7,24.1,29.3,29.5,31.6,31.9,34.6,35.5,61.1,65.4$, $65.7,128.2,128.4,128.6,129.4,129.7,133.6,138.4,140.9$, 166.9, 171.4; MS-electron ionization (EI): $m / z(\%) 453\left(\mathrm{M}^{+}\right.$, 16), 97 (100); high resolution (HR)-MS-EI: $\mathrm{m} / \mathrm{z}\left(\mathrm{M}^{+}\right)$Calcd for $\mathrm{C}_{28} \mathrm{H}_{39} \mathrm{NO}_{4}$ 453.2879, Found 453.2880. The enantiomeric ratios of 3a was determined using HPLC through Daicel CHIRALPAK IC column $(n-\mathrm{Hex} / \mathrm{IPA}=80 / 20,0.5 \mathrm{~mL} / \mathrm{min}, \mathrm{UV}$ $254 \mathrm{~nm}), t_{\mathrm{R}}=15.0 \mathrm{~min}$ and $23.7 \mathrm{~min}, 64 \%$ ee. $[\alpha]_{\mathrm{D}}^{26}-5.7(c=1.12$, $\left.\mathrm{CHCl}_{3}\right)$.

2-(tert-Butoxycarbonylamino)-2-(hydroxymethyl)-4-(4octylphenyl)butyl Benzoate 3b (Entry 8) To a solution of tetraphenylbisoxazoline (L2) $(24 \mathrm{mg}, 0.05 \mathrm{mmol})$ in THF $(2.5 \mathrm{~mL})$ was added $\mathrm{CuCl}_{2}(6.7 \mathrm{mg}, 0.05 \mathrm{mmol})$ and stired at room temperature for $1 \mathrm{~h}$. To the mixture was added THF (5.0 mL), tert-butyl(1-hydroxy-2-(hydroxymethyl)-4-(4octylphenyl)butan-2-yl)carbamate (102 mg, $0.25 \mathrm{mmol}), \mathrm{Et}_{3} \mathrm{~N}$ $(0.37 \mathrm{~mL}, 0.26 \mathrm{mmol})$, benzoyl chloride $(0.23 \mathrm{~mL}, 0.26 \mathrm{mmol})$ were added. After being stirred at room temperature for $24 \mathrm{~h}$, the mixture was poured into water and extracted with EtOAc. The organic layer was washed with brine, dried over $\mathrm{Na}_{2} \mathrm{SO}_{4}$, and concentrated in vacuo. The residue was purified by silica gel column chromatography (hexane-EtOAc) to give $\mathbf{3 b}$ $(67 \mathrm{mg}, 52 \%)$ as a colorless oil.

IR $(\mathrm{KBr})\left(\mathrm{cm}^{-1}\right) \quad 3279,1719,1682 ;{ }^{1} \mathrm{H}-\mathrm{NMR} \quad(400 \mathrm{MHz}$, $\left.\mathrm{CDCl}_{3}\right) \delta: 0.87(\mathrm{t}, J=7.1 \mathrm{~Hz}, 3 \mathrm{H}), 1.20-1.35(\mathrm{~m}, 10 \mathrm{H}), 1.57$ (quin., $J=7.3 \mathrm{~Hz}, 2 \mathrm{H}$ ), 1.98 (ddd, $J=13.7,12.1,5.3 \mathrm{~Hz}, 1 \mathrm{H}$ ), $2.20(\mathrm{ddd}, J=14.0,12.1,5.0 \mathrm{~Hz}, 1 \mathrm{H}), 2.55(\mathrm{t}, J=8.0 \mathrm{~Hz}, 2 \mathrm{H})$, 2.58 (ddd, $J=13.1,13.1,5.0 \mathrm{~Hz}, 1 \mathrm{H}), 2.69$ (ddd, $J=13.1,13.1$, $5.0 \mathrm{~Hz}, 1 \mathrm{H}), 3.78$ (t, $J=5.5 \mathrm{~Hz}, 2 \mathrm{H}), 3.97$ (brs, $1 \mathrm{H}), 4.46$ (d, $J=11.5 \mathrm{~Hz}, 1 \mathrm{H}), 4.58$ (d, $J=11.5 \mathrm{~Hz}, 1 \mathrm{H}), 4.94$ (br s, 1H), 7.07 $(\mathrm{d}, J=8.5 \mathrm{~Hz}, 2 \mathrm{H}), 7.10(\mathrm{~d}, J=8.5 \mathrm{~Hz}, 2 \mathrm{H}), 7.46(\mathrm{t}, J=8.0 \mathrm{~Hz}$, $2 \mathrm{H}), 7.59(\mathrm{tt}, J=6.2,1.4 \mathrm{~Hz}, 1 \mathrm{H}), 8.03(\mathrm{dd}, J=8.5,1.4 \mathrm{~Hz}$, $2 \mathrm{H}) ;{ }^{13} \mathrm{C}-\mathrm{NMR}\left(100 \mathrm{MHz}, \mathrm{CDCl}_{3}\right) \delta: 14.1,22.7,28.3,29.3$, $29.4,29.5,31.6,31.9,35.5,59.0,65.2,65.5,80.2,128.2,128.5$, $129.5,129.8,133.4,138.7,140.7,155.7,166.8$; MS-EI: $m / z$ (\%) $511\left(\mathrm{M}^{+}, 2.5\right), 105$ (100); HR-MS-EI: $m / z\left(\mathrm{M}^{+}\right)$Calcd for $\mathrm{C}_{31} \mathrm{H}_{45} \mathrm{NO}_{5}$ 511.3298, Found 511.3297. The enantiomeric ratios of $\mathbf{3 b}$ was determined using HPLC through Daicel CHIRALPAK IC column ( $n$-Hex/IPA $=80 / 20,0.5 \mathrm{~mL} / \mathrm{min}$, UV $254 \mathrm{~nm}), t_{\mathrm{R}}=39.0 \mathrm{~min}$ and $41.6 \mathrm{~min}, 64 \%$ ee. $[\alpha]_{\mathrm{D}}^{26}-5.0$ $\left(c=1.58, \mathrm{CHCl}_{3}\right)$.

(R)-2-Acetamido-2-(hydroxymethyl)-4-(4-octylphenyl)butyl Acetate $3 \mathrm{c}^{3)}$ (Entry 3) White solid; mp 75-78 $\mathrm{C}$; IR $(\mathrm{KBr})\left(\mathrm{cm}^{-1}\right)$ 3303, 1737, 1631; ${ }^{1} \mathrm{H}-\mathrm{NMR}\left(400 \mathrm{MHz}, \mathrm{CDCl}_{3}\right)$ $\delta: 0.88(\mathrm{t}, J=6.6 \mathrm{~Hz}, 3 \mathrm{H}), 1.20-1.40(\mathrm{~m}, 10 \mathrm{H}), 1.57$ (quin., $J=7.1 \mathrm{~Hz}, 2 \mathrm{H}), 1.85-2.00(\mathrm{~m}, 1 \mathrm{H}), 1.94(\mathrm{~s}, 3 \mathrm{H}), 2.06-2.20$ $(\mathrm{m}, 1 \mathrm{H}), 2.11(\mathrm{~s}, 3 \mathrm{H}), 2.55(\mathrm{t}, J=7.6 \mathrm{~Hz}, 2 \mathrm{H}), 2.50-2.60(\mathrm{~m}$, $1 \mathrm{H}), 2.65$ (ddd, $J=13.5,11.2,5.5 \mathrm{~Hz}, 1 \mathrm{H}), 3.72$ (d, $J=12.1 \mathrm{~Hz}$, $1 \mathrm{H}), 3.77$ (d, $J=12.1 \mathrm{~Hz}, 1 \mathrm{H}), 4.19$ (d, $J=11.5 \mathrm{~Hz}, 1 \mathrm{H}), 4.37$ (d, $J=11.5 \mathrm{~Hz}, 1 \mathrm{H}), 4.62$ (brs, 1H), 5.83 (brs, 1H), 7.09 (s, 4H); ${ }^{13} \mathrm{C}-\mathrm{NMR}\left(100 \mathrm{MHz}, \mathrm{CDCl}_{3}\right) \delta: 14.1,20.9,22.7,24.0,29.2$, 29.3, 29.5, 31.6, 31.9, 34.5, 35.5, 60.7, 65.3, 65.4, 128.2, 128.6, 138.4, 140.8, 171.3, 171.4; MS-EI: $m / z$ (\%) $391\left(\mathrm{M}^{+}, 16\right), 97$ (100); HR-MS-EI: $m / z\left(\mathrm{M}^{+}\right)$Calcd for $\mathrm{C}_{23} \mathrm{H}_{37} \mathrm{NO}_{4}$ 391.2723, Found 391.2715. The enantiomeric ratios of $\mathbf{3 c}$ was determined using HPLC through Daicel CHIRALPAK IC column $\left(\mathrm{CH}_{2} \mathrm{Cl}_{2}\right.$ only, $1.0 \mathrm{~mL} / \mathrm{min}$, UV $254 \mathrm{~nm}$ ), $t_{\mathrm{R}}=39.0 \mathrm{~min}$ and $41.9 \mathrm{~min}, 30 \%$ ee. $[\alpha]_{\mathrm{D}}^{26}-2.0\left(c=1.78, \mathrm{CHCl}_{3}\right)$.

2-(tert-Butoxycarbonylamino)-2-(hydroxymethyl)-4-(4octylphenyl)butyl Acetate 3d (Entry 6) Collorless oil; IR $(\mathrm{KBr})\left(\mathrm{cm}^{-1}\right)$ 3393, 1746, 1719, 1700; ${ }^{1} \mathrm{H}-\mathrm{NMR}(400 \mathrm{MHz}$, $\left.\mathrm{CDCl}_{3}\right) \delta: 0.88(\mathrm{t}, J=6.6 \mathrm{~Hz}, 3 \mathrm{H}), 1.20-1.40(\mathrm{~m}, 10 \mathrm{H}), 1.45$ (s, 9H), 1.58 (quin., $J=7.6 \mathrm{~Hz}, 2 \mathrm{H}$ ), 1.88 (ddd, $J=14.0,12.1$, $5.3 \mathrm{~Hz}, 1 \mathrm{H}$ ), 2.07 (ddd, $J=14.0,12.1,5.0 \mathrm{~Hz}, 1 \mathrm{H}), 2.10$ (s, 3H), $2.48-2.58(\mathrm{~m}, 1 \mathrm{H}), 2.36$ (t, $J=7.6 \mathrm{~Hz}, 2 \mathrm{H}), 2.63$ (ddd, $J=13.5$, $11.2,5.5 \mathrm{~Hz}, 1 \mathrm{H}), 3.68(\mathrm{dd}, J=11.9,6.2 \mathrm{~Hz}, 1 \mathrm{H}), 3.74$ (dd, $J=11.9,6.2 \mathrm{~Hz}, 1 \mathrm{H}), 3.93$ (s, 1H), 4.16 (d, $J=11.2 \mathrm{~Hz}, 1 \mathrm{H}), 4.34$ (d, $J=11.2 \mathrm{~Hz}, 1 \mathrm{H}), 4.87$ (brs, 1H), 7.09 (s, 4H); ${ }^{13} \mathrm{C}-\mathrm{NMR}$ $\left(100 \mathrm{MHz}, \mathrm{CDCl}_{3}\right) \delta: 14.1,20.9,22.7,28.3,29.1,29.3,29.4$, $31.6,31.9,34.9,35.5,58.6,65.3,80.2,128.2,128.5,138.7$, 140.7, 155.7, 171.2; MS-EI: $\mathrm{m} / z$ (\%) $449\left(\mathrm{M}^{+}, 4.8\right), 318$ (100); HR-MS-EI: $m / z\left(\mathrm{M}^{+}\right)$Calcd for $\mathrm{C}_{26} \mathrm{H}_{43} \mathrm{NO}_{5}$ 449.3141, Found 449.3143. The enantiomeric ratios of $\mathbf{3 d}$ was determined using HPLC through Daicel CHIRALPAK IC column $\left(\mathrm{CH}_{2} \mathrm{Cl}_{2}\right.$ only, $1.0 \mathrm{~mL} / \mathrm{min}, \mathrm{UV} 254 \mathrm{~nm}), t_{\mathrm{R}}=9.1 \mathrm{~min}$ and $26.7 \mathrm{~min}, 8 \%$ ee. $[\alpha]_{\mathrm{D}}^{26}-0.2\left(c=3.76, \mathrm{CHCl}_{3}\right)$.

\section{References}

1) Hamada M., Adachi K., Hikawa H., Yokoyama Y., Chem. Pharm. Bull., 60, 1395-1398 (2012).

2) Hamada M., Nakamura M., Kiuchi M., Marukawa K., Tomatsu A., Shimano K., Sato N., Sugahara K., Asayama M., Takagi K., Adachi K., J. Med. Chem., 53, 3154-3168 (2010).

3) Kiuchi M., Adachi K., Tomatsu A., Chino M., Takeda S., Tanaka Y. Maeda Y., Sato N., Mitsutomi N., Sugahara K., Chiba K., Bioorg. Med. Chem., 13, 425-432 (2005).

4) Lu X., Sun C., Valentine W. J., Shuyu E., Liu J., Tigyi G., Bittman R., J. Org. Chem., 74, 3192-3195 (2009).

5) Lu X., Bittman R., Tetrahedron Lett., 47, 825-827 (2006).

6) Jiang X., Gong B., Prasad K., Repic O., Org. Process Res. Dev., 12, 1164-1169 (2008).

7) Hale J. J., Yan L., Neway W. E., Hajdu R., Bergstrom J. D., Milligan J. A., Shei G.-J., Chrebet G. L., Thornton R. A., Card D., Rosenbach M., HughRosen, Mandala S., Bioorg. Med. Chem., 12, 4803-4807 (2004).

8) Albert R., Hinterding K., Brinkmann V., Guerini D., MullerHartwieg C., Knecht H., Simeon C., Streiff M., Wagner T., Welzenbach K., Zecri F., Zollinger M., Cooke N., Francotte E., J. Med. Chem., 48, 5373-5377 (2005).

9) Oriyama T., Imai K., Sano T., Hosoya T., Tetrahedron Lett., 39, 3529-3532 (1998)

10) Matsumura Y., Maki T., Murakami S., Onomura O., J. Am. Chem. Soc., 125, 2052-2053 (2003).

11) Mizuta S., Sadamori M., Fujimoto T., Yamamoto I., Angew. Chem. Int. Ed., 42, 3383-3385 (2003).

12) Oriyama T., Taguchi H., Terakado D., Sano T., Chem. Lett., 2002, 26-27 (2002)

13) Lewis C. A., Sculimbrene B. R., Xu Y., Miller S. J., Org. Lett., 7, 3021-3023 (2005).

14) Mitsuda M., Tanaka T., Tanaka T., Demizu Y., Onomura O., Matsumura Y., Tetrahedron Lett., 47, 8073-8077 (2006).

15) Hong M. S., Kim T. W., Jung B., Kang S. H., Chem. Eur. J., 14, 3290-3296 (2008).

16) Honjo T., Nakao M., Sano S., Shiro M., Yamaguchi K., Sei Y., Nagao Y., Org. Lett., 9, 509-512 (2007).

17) Jung B., Hong M. S., Kang S. H., Angew. Chem. Int. Ed., 46, $2616-$ 2618 (2007).

18) Hikawa H., Hamada M., Yokoyama Y., Azumaya I., RSC Adv., 4, 23131-23136 (2014). 
19) Mei T.-W., Luo Y., Feng X.-J., Lu W., Yang B., Tetrahedron, 69, 2927-2932 (2013).

20) Ko R. Y. Y., Chu J. C. K., Chiu P., Tetrahedron, 67, 2542-2547 (2011).

21) Zivkovic A., Stark H., Tetrahedron Lett., 51, 3769-3771 (2010).

22) Li Z., Bittman R., J. Org. Chem., 72, 8376-8382 (2007).

23) Kim S., Lee H., Lee M., Lee T., Synthesis, 2006, 753-755 (2006).

24) Kandagatla B., Prasada Raju V. V. N. K. V., Kumar N. S., Reddy G. M., Srinivas K., Iqbal J., Bandichhor R., Oruganti S., RSC Adv., 3, 9687-9689 (2013).
25) Nakayama S., Uto Y., Tanimoto K., Okuno Y., Sasaki Y., Nagasawa H., Nakata E., Arai K., Momose K., Fujita T., Hashimoto T., Okamoto Y., Asakawa Y., Goto S., Hori H., Bioorg. Med. Chem., 16, 7705-7714 (2008).

26) Ma B., Guckian K. M., Lin E. Y.-S., Lee W.-C., Scott D., Kumaravel G., Macdonald T. L., Lynch K. R., Black C., Chollate S., Hahm K., Hetu G., Jin P., Luo Y., Rohde E., Rossomando A., Scannevin R., Wang J., Yang C., Bioorg. Med. Chem. Lett., 20, 2264-2269 (2010). 